\title{
The Contribution of Internal and External Assets of Resilience in Explaining Peer Violence
}

\author{
Matea Belošević1, Martina Ferić ${ }^{2}$, Ivana Mitrić3 \\ Laboratory for prevention research (PrevLab), Department of Behaviour Disorders, Faculty of Education and \\ Rehabilitation Sciences, University of Zagreb
}

\begin{abstract}
.
The Laboratory for Prevention Research, Department of Behavioral Disorders, ERF-UNIZG is the leader of the project "Positive Development of Adolescents in Croatia", which aimed to study indicators of positive development, risk behavior and mental health of adolescents. Previous studies have shown that resilience is associated with positive youth development and less participation in different risk behaviors. This paper aims to determine the contribution of internal and external assets of resilience to the explanation of peer violence of the adolescents. The study involved 1,647 adolescents from the county from North of Croatia (46\% girls and 52\% boys, aged 14 to 19 years), which is $30 \%$ of all enrolled high school students in the project. To answer our research question, CTC Youth Survey (modified by Mihić, Novak and Bašić, 2011) and a modified version of the Resilience and Youth Development Module (California Department of Education, 1997) were examined. Logistic regression analysis was used to test the hypothesis of this paper. In general, the results showed that predictors that reduce the possibility of peer violence are higher levels of cooperation \& communication, self-efficacy, high-expectation pro-social peers and caring relationship in peer and family environment. On the other hand, predictors that increase the possibility of peer violence are female gender and a higher level of empathy. These results suggest that attention to assets of resilience which can help youth navigate adolescence in healthy ways - is promising for development and implementation of prevention programs for peer violence.
\end{abstract}

Keywords: adolescence; age; gender; positive youth development; risk behaviour 